\title{
Design Optimization of GaN-based VCSELs
}

\author{
Joachim Piprek ${ }^{1}$ and Zhan-Ming (Simon) $\mathrm{Li}^{2}$ \\ ${ }^{1}$ NUSOD Institute LLC, Newark, DE 19714-7204, United States, E-mail: piprek@ieee.org \\ ${ }^{2}$ Crosslight Software Inc., 121-3989 Henning Drive, Burnaby, BC V5C 6P8 Canada
}

\begin{abstract}
We analyze recently manufactured designs of electrically pumped GaN-based vertical-cavity surface-emitting lasers (VCSELs) using advanced laser simulation software. Thick quantum wells are found to allow for the almost complete elimination of the built-in quantum well polarization field. The simulations also reveal several performance limiting effects, e.g., current crowding and electron leakage. Design optimization options are proposed and discussed.
\end{abstract}

\section{INTRODUCTION}

GaN-based vertical-cavity surface-emitting lasers (VCSELs) are expected to exhibit several advantages over their edge-emitting counterparts, including lower manufacturing costs, circular output beams, and longer lifetime. In contrast to the great success of GaN-based edge-emitting lasers in recent years, GaN-VCSELs still face significant challenges. Electrically pumped devices have been demonstrated only recently and they exhibit severe performance restrictions. ${ }^{1,2}$

Based on our previous experience with VCSEL design and simulation, ${ }^{3}$ we here analyze these recently manufactured GaN-VCSELs using advanced laser simulation software. ${ }^{4}$ The simulation self-consistently combines carrier transport, photon emission, and multimode optical wave guiding. Self-heating is neglected here as we investigate pulsed operation at room temperature in this paper. The transport model includes drift and diffusion of electrons and holes, Fermi statistics, built-in polarization and thermionic emission at hetero-interfaces, as well as defect-related Shockley-Read-Hall recombination of carriers. For the quantum wells, Schrödinger and Poisson equations are solved iteratively at every bias point to account for the Quantum-Confined Stark Effect. Stimulated and spontaneous emission of photons within the quantum well is calculated based on the wurtzite energy band structure. A similar model was previously used to study GaN-based edgeemitting lasers, resulting in excellent agreement between measurements and simulations. ${ }^{5}$

\section{460nm VCSEL DESIGN}

The first design investigated is tailored for light emission at $\lambda=460 \mathrm{~nm}$. ${ }^{1}$ The $5-\lambda$-cavity is sandwiched between a 29-period $\mathrm{AlN} / \mathrm{GaN}$ distributed Bragg reflector (DBR) on the n-doped side and an 8-period $\mathrm{Ta}_{2} \mathrm{O}_{5} / \mathrm{SiO}_{2}$ dielectric $\mathrm{DBR}$ on the p-side (Fig. 1). The multi-quantum well (MQW) active region comprises $10 \mathrm{InGaN}$ quantum wells (each $2.5 \mathrm{~nm}$ thick) that are separated by $7.5 \mathrm{~nm}$ GaN barriers. The current is injected through a $240 \mathrm{~nm}$ thick indium tin oxide (ITO) layer on the $\mathrm{p}$ - side ( $5 \mu \mathrm{m}$ aperture radius) and an intra-cavity ring contact on the n-side. This VCSEL shows continuous-wave (CW) lasing only at very low temperatures $(77 \mathrm{~K}){ }^{1}$

As both the cavity wavelength and the QW gain spectrum red-shift with rising temperature, a careful adjustment of the QW gain spectrum is required to achieve lasing at room temperature. ${ }^{6}$ Our simulation adjusts the QW composition to move the room-temperature gain peak into close proximity of the cavity wavelength. However, the MQW active region is wider than the peak of the standing optical wave and some of the quantum wells do not contribute to the lasing process (Fig. 1). The thick ITO layer causes significant optical loss (1.4\%). This loss can be reduced by using a thinner ITO layer that is placed at the node of the standing wave. ${ }^{3}$

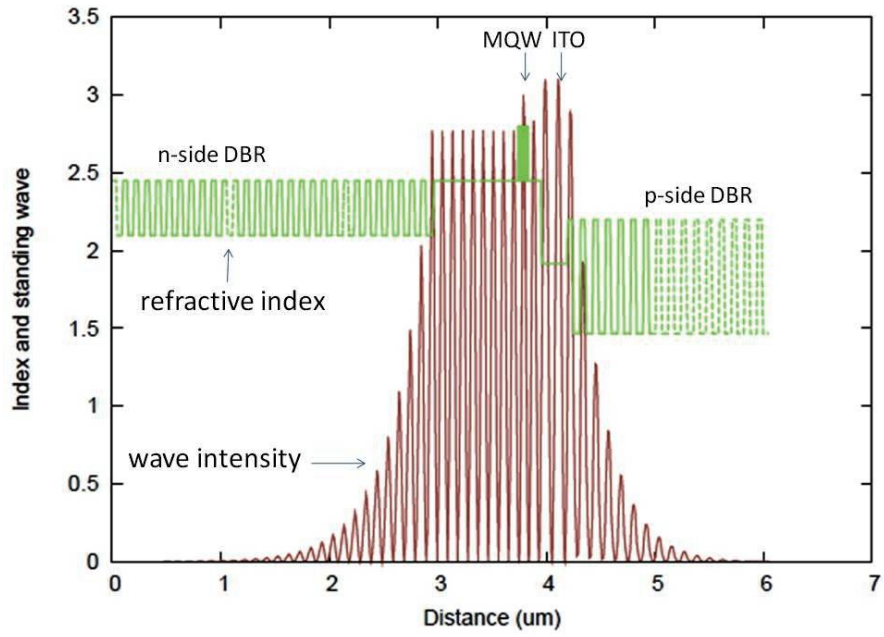

Fig. 1 Vertical index profile and optical intensity of the 460nm VCSEL. ${ }^{1}$

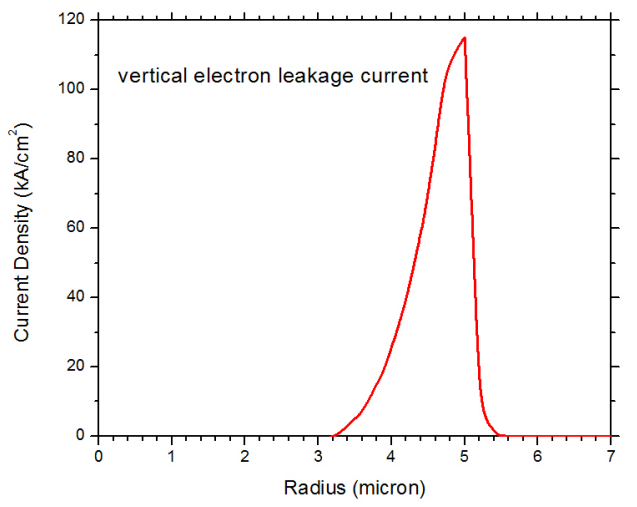

Fig. 2 Lateral electron current profile within the p-GaN layer above the MQW calculated at $25 \mathrm{~mA}$ injection current. 
The calculated electron current profile within the $\mathrm{p}$ GaN layer is plotted in Fig. 2. The sharp peak of the electron current at the ITO aperture radius $(\mathrm{r}=5 \mu \mathrm{m})$ not only reveals significant electron leakage into the $\mathrm{p}-\mathrm{GaN}$ layer, but also strong current crowding. The electron leakage severely limits the quantum efficiency of the laser. Electron overflow can be reduced by inserting an $\mathrm{AlGaN}$ electron stopper layer above the MQW as it is common with GaN-based edge-emitting lasers. ${ }^{5}$ Such AlGaN layer also reduces the current crowding as it enforces a more uniform hole injection into the MQW. ${ }^{3}$

\section{410nm VCSEL DESIGN}

The second design investigated is tailored for light emission at $\lambda=410 \mathrm{~nm}^{2}{ }^{2}$ The $7-\lambda$-cavity is sandwiched between an 11.5-period $\mathrm{Nb}_{2} \mathrm{O}_{5} / \mathrm{SiO}_{2}$ DBR on the p-side and a 7-period $\mathrm{Nb}_{2} \mathrm{O}_{5} / \mathrm{SiO}_{2}$ DBR on the n-side (Fig. 3). The active region comprises two $9 \mathrm{~nm}$ thick InGaN quantum wells separated by a $13 \mathrm{~nm} \mathrm{GaN}$ barrier. The current is injected through a $50 \mathrm{~nm}$ thick ITO layer on the p-side (4 $4 \mathrm{~m}$ aperture radius) and an intra-cavity ring contact on the n-side. This GaN-VCSEL is the first to show room-temperature $\mathrm{CW}$ lasing. ${ }^{2}$

The optical simulation results in Fig. 3 show that the ITO layer is well positioned near the node of the standing wave, thereby reducing the optical loss. The two quantum wells are near the peak of the standing wave with a gain enhancement factor of 1.6. The strong increase in QW thickness leads to multiple quantum levels occupied under lasing operation (see Figs. 4 \& 5). The QW carriers are able to effectively screen the built-in polarization resulting in almost flat-band conditions (Fig. 4). This overcomes the detrimental effect of built-in polarization observed with thinner quantum wells. ${ }^{3}$

However, also for this improved VCSEL design, our simulations reveal current crowding at the ITO aperture as well as strong electron leakage into the $\mathrm{p}-\mathrm{GaN}$ layer (similar to Fig. 2). The introduction of an $\mathrm{AlGaN}$ electron stopper layer is expected to improve the device performance significantly.

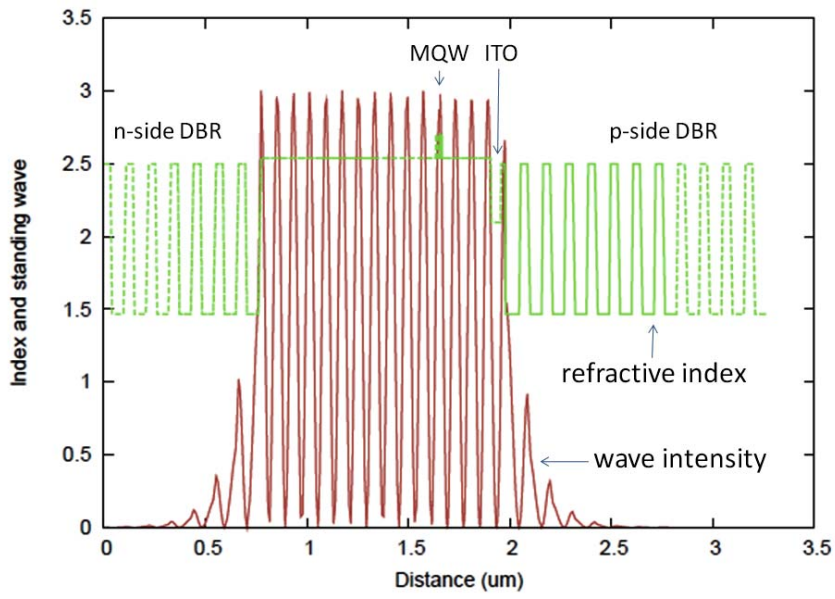

Fig. 3 Vertical index profile and optical intensity of the 410 nm VCSEL. ${ }^{2}$

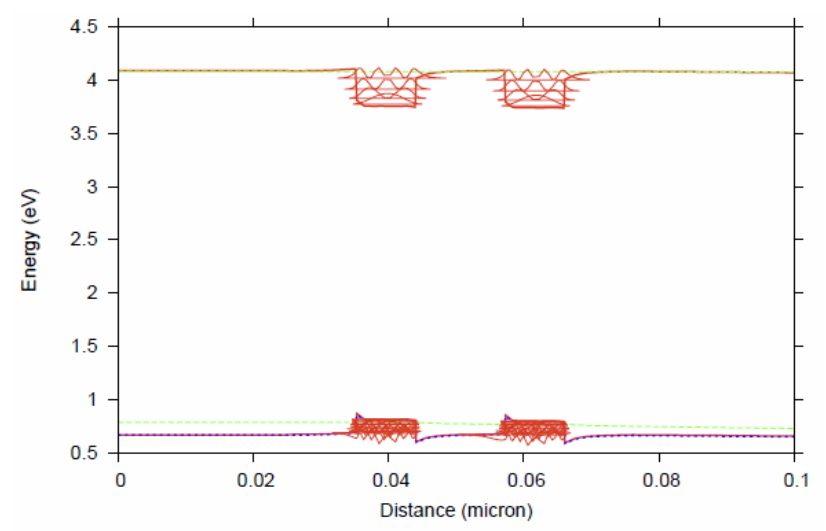

Fig. 4 Energy band diagram of the two quantum wells above lasing threshold including quantum levels and wave functions (green dashed lines - quasi Fermi levels).

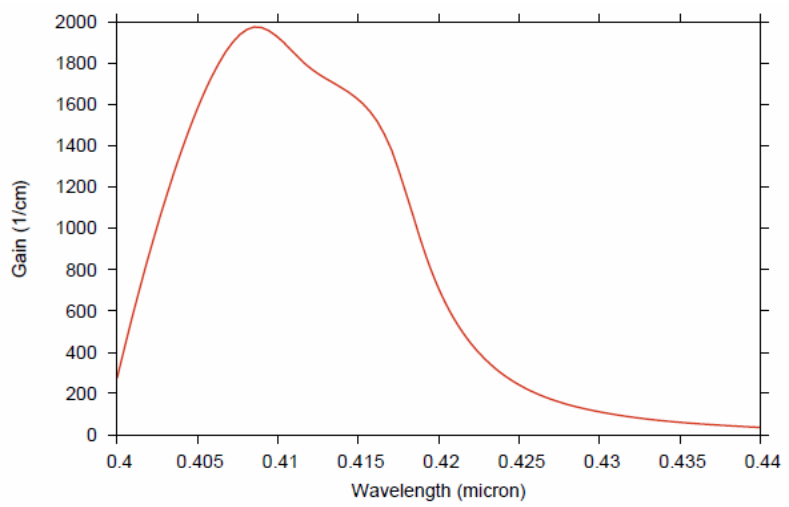

Fig. 5 Quantum well gain spectrum from multiple quantum levels in Fig. 4 calculated at $12 \mathrm{~mA}$ injection current.

\section{REFERENCES}

${ }^{1}$ Tien-Chang Lu, Chih-Chiang Kao, Hao-Chung Kuo, GenSheng Huang, and Shing-Chung Wang, "CW lasing of current injection blue GaN-based vertical cavity surface emitting laser, “ Appl. Phys. Lett. Vol. 92, 141102 (2008).

${ }^{2} \mathrm{Yu}$ Higuchi, Kunimichi Omae, Hiroaki Matsumura, and Takashi Mukai, "Room-Temperature CW Lasing of a GaNBased Vertical-Cavity Surface-Emitting Laser by Current Injection, “ Appl. Phys. Expr. Vol. 1, 121102 (2008).

3 J. Piprek, Z.-M. Li, R. Farrell, S. P. DenBaars, and S. Nakamura, "Electronic Properties of InGaN/GaN VerticalCavity Lasers," Ch. 19 in Nitride Semiconductor DevicesPrinciples and Simulation, ed. J. Piprek, Wiley-VCH, Weinheim 2007.

${ }_{5}^{4}$ PICS3D by Crosslight Software, 2009.

5 J. Piprek and S. Nakamura, "Physics of high-power InGaN/GaN lasers," IEE Proc.-Optoelectron., vol. 149, p. 145, 2002.

${ }^{6}$ S. Rapp, J. Piprek, K. Streubel, J. Andre, and J. Wallin, "Temperature sensitivity of $1.54 \mu \mathrm{m}$ Vertical-Cavity Lasers with an InP-based Bragg Reflector,” J. Quantum Electr., vol. 33, p. 1839 (1997). 